\title{
Laparoscopic rectopexy for rectal prolapse
}

Vikal Chandra Shakya

Department of Surgery, Civil Service Hospital, Minbhawan, Kathmandu

Correspondence: Vikal Chandra Shakya; Specialist, Department of Surgery, Civil Service Hospital, Minbhawan, Kathmandu

Email: vikalcsh@yahoo.com .

\begin{abstract}
Introduction and Objective: Rectal prolapse surgery has been considered advanced laparoscopic surgery; hence it has mostly been performed by open surgery. However, with advancement, laparoscopic rectopexy can be feasible in patients with rectal prolapse.
\end{abstract}

Materials and Methods: This study was done in patients presenting to Surgery Department of Civil Service Hospital who underwent laparoscopic rectopexy from January 2013 to November 2015

Results: There were 8 patients ( 4 females and 4 males). The mean operative time was 160+49 minutes. The mean postoperative stay was $3+1.21$ days. There was no conversion. One child underwent suture rectopexy, whereas adults underwent posterior mesh rectopexy. No patients needed blood transfusion. Postoperatively one patient has constipation, and she needed occasional laxatives.

Conclusion: Laparoscopic rectopexy is a minimally invasive advanced procedure with all the advantages of laparoscopy. With further experience operative time could be reduced.

Key words: rectal prolapse; rectopexy; mesh 